\title{
Microfabricated photoplastic cantilever with integrated photoplastic/carbon based piezoresistive strain sensor
}

\author{
Gammelgaard, Lauge; Rasmussen, Peter Andreas; Calleja, M.; Vettiger, P.; Boisen, Anja
}

Published in:

Applied Physics Letters

Link to article, DOI:

$10.1063 / 1.2186396$

Publication date:

2006

Document Version

Publisher's PDF, also known as Version of record

Link back to DTU Orbit

Citation (APA):

Gammelgaard, L., Rasmussen, P. A., Calleja, M., Vettiger, P., \& Boisen, A. (2006). Microfabricated photoplastic cantilever with integrated photoplastic/carbon based piezoresistive strain sensor. Applied Physics Letters, 88(11), 113508. https://doi.org/10.1063/1.2186396

\section{General rights}

Copyright and moral rights for the publications made accessible in the public portal are retained by the authors and/or other copyright owners and it is a condition of accessing publications that users recognise and abide by the legal requirements associated with these rights.

- Users may download and print one copy of any publication from the public portal for the purpose of private study or research.

- You may not further distribute the material or use it for any profit-making activity or commercial gain

- You may freely distribute the URL identifying the publication in the public portal 


\title{
Microfabricated photoplastic cantilever with integrated photoplastic/carbon based piezoresistive strain sensor
}

\author{
L. Gammelgaard, ${ }^{\text {a) }}$ P. A. Rasmussen, M. Calleja, P. Vettiger, and A. Boisen \\ Department of Micro- and Nanotechnology, Technical University of Denmark, DK-2800 Kgs. Lyngby, \\ Denmark
}

(Received 26 July 2005; accepted 9 February 2006; published online 15 March 2006)

\begin{abstract}
We present an SU-8 micrometer sized cantilever strain sensor with an integrated piezoresistor made of a conductive composite of SU-8 polymer and carbon black particles. The composite has been developed using ultrasonic mixing. Cleanroom processing of the polymer composite has been investigated and it has been shown that it is possible to pattern the composite by standard UV photolithography. The composite material has been integrated into an SU-8 microcantilever and the polymer composite has been demonstrated to be piezoresistive with gauge factors around 15-20. Since SU-8 is much softer than silicon and the gauge factor of the composite material is relatively high, this polymer based strain sensor is more sensitive than a similar silicon based cantilever sensor. (C) 2006 American Institute of Physics. [DOI: 10.1063/1.2186396]
\end{abstract}

Micromachined cantilevers are often used for atomic force microscope imaging. Today microcantilevers are used for a variety of applications including biological and chemical measurements. ${ }^{-3}$ We find that cantilevers with integrated readout offer some advantages as compared to the typical laser leverage readout when it comes to making compact systems and obtaining easily accessible signals (no aligning, no light-to-voltage conversion). Lately, several highly sensitive cantilever sensors with integrated piezoresistive readout have been developed. ${ }^{4-7}$

The working principle is that a change in surface stress makes the cantilever deflect which again gives rise to a strain in the integrated piezoresistor. The change of resistance in the piezoresistor can then be measured.

Cantilever sensors have the possibility to perform very local and high-resolution measurements. For example measurements of DNA hybridization or antigen/antibody recognition can be made in microliquid channels establishing a basis for point of care applications.

Cantilevers are normally made of silicon which has a high Young's modulus and a cantilever made of a softer material would be more sensitive for static deflection measurements. This can be seen from the following equation describing the force sensitivity of a rectangular cantilever

$$
\frac{\Delta R}{R}=12 \frac{K}{E} \frac{l d}{w t^{3}} F,
$$

where $\Delta R / R$ is the relative change in resistance and $K$ is the gauge factor of the piezoresistor respectively, $E$ is Young's modulus, $l, w, t$ are the length, width, and thickness of the cantilever, $d$ is the distance from the piezoresistor to the neutral axis, and $F$ is the force applied at the apex of the cantilever. Apart from the cantilever dimensions it is the relation between the gauge factor and Young's modulus of a material that determines the sensitivity of the cantilever sensor.

Recently, polymers with a much lower Young's modulus than that of silicon have been used for fabricating cantilevers as a substitute material for silicon. ${ }^{8}$ However, these materials

\footnotetext{
${ }^{a)}$ Electronic mail: lag@mic.dtu.dk
}

do not have any piezoresistive properties. Previously, we have developed SU-8 cantilevers with integrated Au resistors that have a gauge factor of approximately $2 .{ }^{9,10}$ In these cantilevers the low gauge factor of the Au resistors is limiting the sensitivity of the cantilever sensor.

In this letter we present the development of a micromachined SU-8 cantilever with integrated readout based on a piezoresistive SU-8/carbon composite material. The final device is shown in Fig. 1.

The cantilever consists of two SU-8 layers encapsulating the SU-8/carbon composite material and Au electrodes. The thickness of the cantilever is approximately $7 \mu \mathrm{m}$. The cantilever is placed on an SU-8 support structure approximately $200 \mu \mathrm{m}$ thick (see Fig. 2).

Percolation theory describes the conductivity of polymer composite materials based on a conducting network of randomly dispersed conducting particles in the insulating polymer matrix. ${ }^{11}$ The resistivity of the composite material with carbon particles above the percolation threshold can generally be modelled by the power law relation

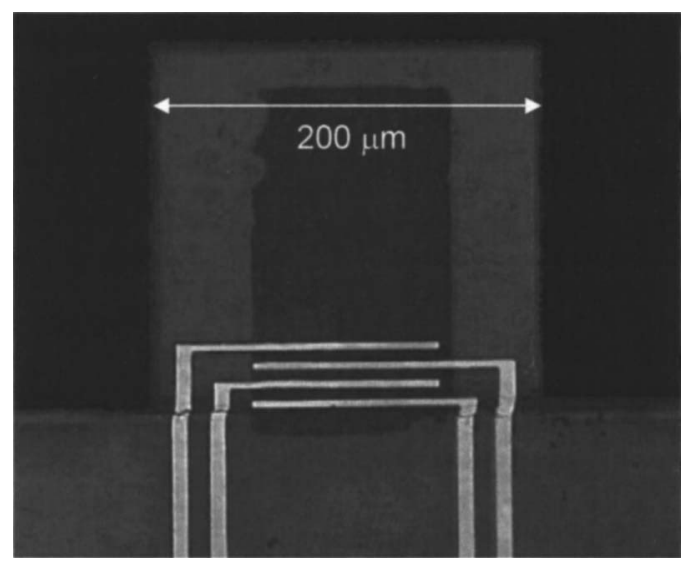

FIG. 1. Optical image of a micromachined SU-8 cantilever with integrated $\mathrm{SU}-8 /$ carbon piezoresistor and four Au electrodes seen from the top. The cantilever is $200 \mu \mathrm{m}$ long, $200 \mu \mathrm{m}$ wide, and approximately $7 \mu \mathrm{m}$ thick. 


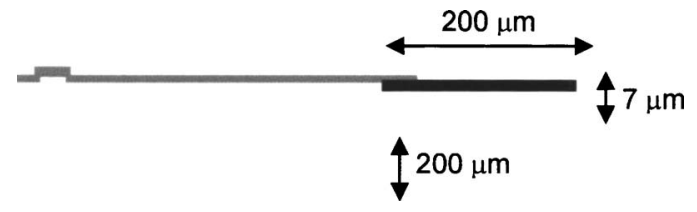

FIG. 2. Schematic drawing of the cantilever. SU-8 support structure and SU-8 cantilever layers (light gray). Piezoresistive composite material (black), and gold electrodes (dark gray).

$$
\rho=\rho_{o}\left(\frac{P-P_{c}}{1-P_{c}}\right)^{-1}
$$

where $\rho$ is the resistivity, $\rho_{0}$ is the resistivity scale factor, $P$ is the carbon concentration, $P_{c}$ is the carbon concentration at the percolation threshold, and $t$ is the so called critical exponent which is connected to the dimensionality of the system. ${ }^{12}$ Both $\rho_{o}$ and $t$ are usually used as fitting parameters in experimental work.

The polymer composite is fabricated by ultrasonic mixing of SU-8 2002 and the carbon particles Conductor 975 Ultra. ${ }^{13}$ Conductex 975 Ultra is a high structure carbon black with an average particle diameter of $21 \mathrm{~nm}$ and is highly conductive. This composite material can be spun on wafers and structured by standard UV lithography and is compatible with all necessary SU-8 clean room processes. It can form thin film layers of less than $1.5 \mu \mathrm{m}$ thickness and structures of $10 \times 10 \mu \mathrm{m}$ are possible to achieve with the existing fabrication process.

The composite samples are prepared by placing a high power ultrasonic source directly into the SU-8 with the added carbon particles. During the mixing the samples are cooled down to avoid evaporation of the SU-8 solvent cyclopentanone.

The resistivity as a function of the carbon filler (weight percent) is shown in Fig. 3. These measurements were made on some special test structures. All resistivity measurements are made with a Keithley 2000 using a simple standard twoprobe method. The test structures are made on Si wafers with an oxide layer on top. Then, Au electrodes are structured on

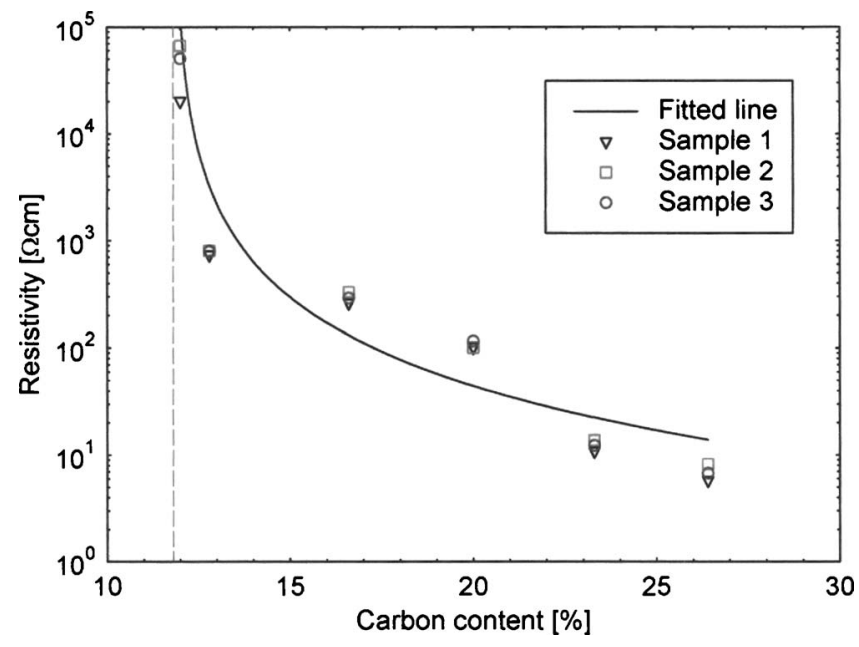

FIG. 3. The resistivity as a function of carbon filler measured on three samples with a standard two-point measurement. The resistance of the composites with less than $12 \%$ carbon particles were out of the scale (of the Keithley 2000) used for these measurements indicating that all of them were insulating. A power law fit is also shown indicating agreement with percolation theory.

Downloaded 16 Jun 2010 to 192.38.67.112. Redistribution subject to AlP license or copyright; see http://apl.aip.org/apl/copyright.jsp

the insulating oxide in order to establish a good contact to the $1.4 \mu \mathrm{m}$ thick composite layer that is deposited on top of the Au electrodes.

The percolation threshold was determined to be close to $12 \%$ (vertical dashed line). A polymer composite with a carbon content of approximately $16 \%$ was chosen for the fabrication of the cantilever sensor. This was done in order to operate well above the percolation threshold such that large fluctuations in the resistance were avoided. ${ }^{14,15}$ At the same time the composite should have a low resistance in order to minimise noise and the gauge factor should be maximized. In Fig. 3 the conducting properties of the SU-8/carbon composite material with different amounts of carbon content is fitted to a power law relation like Eq. (2). The measured resistivity of the composite material seems to indicate a good agreement with percolation theory by showing a very sudden rise in resistivity by several orders of magnitude.

The developed cantilever strain sensor with the integrated piezoresistive SU-8/carbon composite is fabricated by the following process sequence. First a release layer of $\mathrm{Cr} / \mathrm{Au} / \mathrm{Cr}$ with thickness of 50/500/500 $\AA$, respectively, is deposited on a $4 \mathrm{in}$. Si wafer. Then, a thin layer of $800 \mathrm{~nm}$ SU-8 is deposited and structured to use as the topside of the cantilever. Next a $500 \mathrm{~nm}$ layer of $\mathrm{Au}$ is $e$-beam evaporated on top of a $1.5 \mu \mathrm{m}$ thick AZ-5214 photoresist pattern and the gold electrodes are structured with a lift-off process. A $1.4 \mu \mathrm{m}$ layer of the SU-8/carbon composite material is spun on top of the Au wires and structured by UV lithography. To encapsulate the piezoresistor a $4.0 \mu \mathrm{m}$ thick SU-8 layer for the backside of the cantilever is deposited - at the same time this layer roughly determines the distance from the piezoresistor to the neutral axis in the cantilever. Finally, the support structure consistijng of a $200 \mu \mathrm{m}$ thick SU-8 layer is deposited and structured by UV lithography and the chips are released from the wafer in a Cretch. ${ }^{16}$

The force sensitivity of the cantilever sensors fabricated by this process has been determined by deflecting the cantilever. The results can be seen in Fig. 4. The cantilever was deflected with a sharp needle controlled by a micrometer screw in steps of $5 \mu \mathrm{m}$ while the resistance was measured with a Keithley 2000. The accuracy of these steps is within $\pm 1 \mu \mathrm{m}$ on the micrometer screw, but the tip of the needle will slide on the cantilever surface at large deflections giving a too low deflection. How much is difficult to estimate though but these things combined could explain some of the irregularities on the curve. The electrical contacts to the chip were made with a probe station. At first the cantilever was left alone to check if the signal was stable, then it was deflected and then slowly released again. This gave the first peak in Fig. 4. This was repeated (second peak) except that the needle was moved away from the cantilever after the full deflection distance of $50 \mu \mathrm{m}$. The change in base signal seen could be due to breaking of some of the conducting pathways in the composite or drift in the measurement setup.

The slope of the three steps: deflection, release, and second deflection are plotted in Fig. 5. This gives a value for the gauge factor $K$ between 15 and 20 for the SU-8/carbon polymer composite material, see Eq. (1). Since the calculation of the gauge factor is based on a simple model and at the same time very dependent on the exact location of the micrometer screw during the deflection it leaves an error margin of at least $15 \%$. 


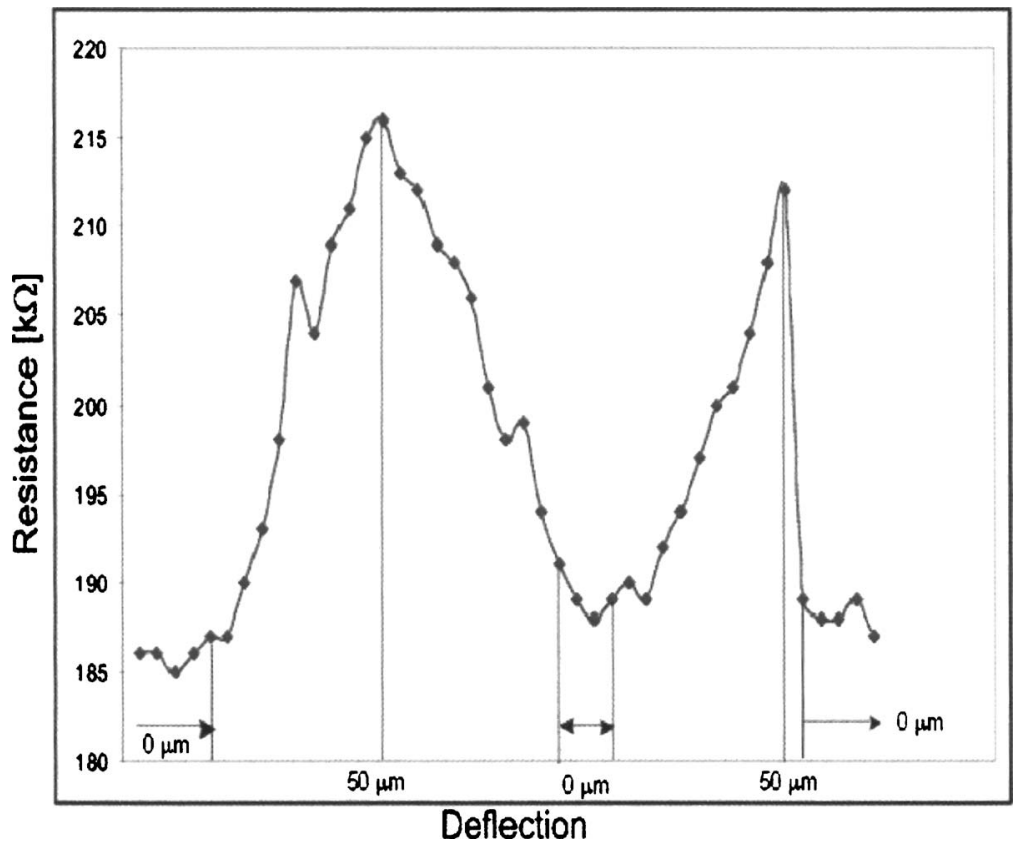

FIG. 4. Repeated endpoint deflection of the cantilever with a length and width of $200 \mu \mathrm{m}$ and a total thickness of $7 \mu \mathrm{m}$. The needle used for bending the cantilever is placed approximately $20 \mu \mathrm{m}$ from the tip of the cantilever. The resistance is measured as a function of the cantilever deflection in steps of $5 \mu \mathrm{m}$. The sudden drop in resistance seen at the second peak is due to the needle being removed from the cantilever.

Young's modulus of SU-8 is approximately $5 \mathrm{GPa}^{17}$. Together with a gauge factor of the composite piezoresistor of 15-20 when integrated in an SU-8 cantilever this gives theoretically a material that is $5-10$ times more force sensitive than a silicon based cantilever with integrated piezoresistive readout if all dimensions of resistor and cantilever are equal. ${ }^{6}$

In conclusion, a micromachined cantilever with integrated readout based on a piezoresistive polymer composite of SU-8 and carbon black particles has been developed. This new piezoresistive polymer composite has been demonstrated to have a gauge factor of approximately $15-20$, which is much higher than alternative non silicon-based materials such as Au. At the same time the composite has a much lower Young's modulus than $\mathrm{Au}$, it can be structured by standard UV lithography and can form a thin film less than $1.5 \mu \mathrm{m}$ thick.

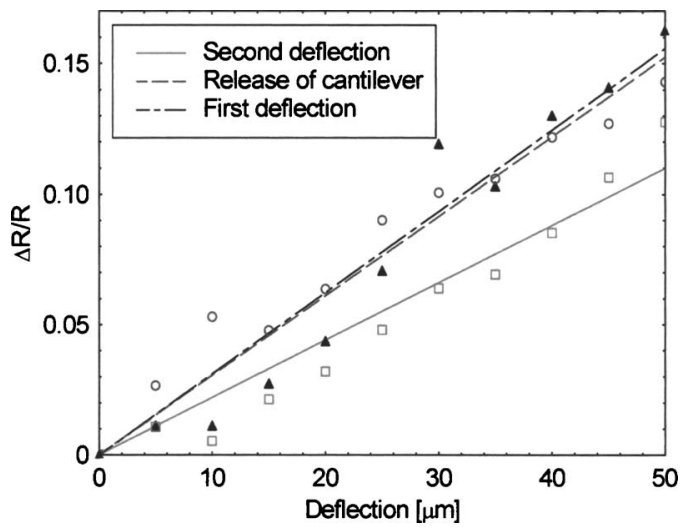

FIG. 5. The relative change in resistance as a function of the cantilever deflection. The three lines represent the three steps in the deflection experiment, deflection, release, and second deflection. The cantilever shows no sign of fatigue or plastic deformation even after several runs.
Potentially this polymer composite can lead to cheap, fast, and flexible processing. The material has shown a high sensitivity that possibly with further work could be increased and it has a broad range of possible application also within other areas than cantilever sensors, like polymer contacts, pressure sensors, harsh environment fluidic electrodes, etc.

${ }^{1}$ H. G. Craighead, Science 290, 1532 (2000).

${ }^{2}$ J. Fritz, M. K. Baller, H. P. Lang, H. Rothuizen, P. Vettiger, E. Meyer, H. J. Guntherodt, Ch. Gerber, and J. W. Gimzewski, Science 288, 316 (2000).

${ }^{3}$ R. Raiteri, M. Grattarola, H.-J. Butt, and P. Skládal, Sens. Actuators B 79, 115 (2001)

${ }^{4}$ A. Boisen, J. Thaysen, H. Jensenius, and O. Hansen, Ultramicroscopy 82, 11 (2000).

${ }^{5}$ Y. Su, A. G. R. Evans, A. Brunnschweiler, and G. Ensell, J. Micromech. Microeng. 12, 780 (2002).

${ }^{6}$ P. A. Rasmussen, PhD thesis, DTU, Lyngby, 2003.

${ }^{7}$ M. Tortonese, H. Yamada, R. C. Barrett, and C. F. Quate, Transducers, 448 (1991).

${ }^{8}$ G. Genolet, J. Brugger, M. Despont, U. Drechsler, P. Vettiger, N. F. Rooij, and D. Anselmetti, Rev. Sci. Instrum. 70, 2398 (1999).

${ }^{9}$ M. Calleja, P. Rasmussen, A. Johansson, and A. Boisen, Proc. SPIE 5116, 314 (2003).

${ }^{10}$ J. Thaysen, A. D. Yalçinkaya, P. Vettiger, and A. Menon, J. Phys. D 35, 2698 (2002)

${ }^{11}$ L. Flandin, A. Chang, S. Nazarenko, and A. Hiltner, J. Appl. Polym. Sci. 76, 894 (2000).

${ }^{12}$ P. R. King, S. V. Buldyrev, N. V. Dokholyan, S. Havlin, E. Lopez, G. Paul, and H. E. Stanley 〈http://dokhlab.unc.edu/papers/kbdhlps-dialog02.pdf〉

${ }^{13}$ Produced by Colombian Chemicals Company $\langle$ http://www.columbianchemicals.com〉

${ }^{14}$ M. B. Heaney, Phys. Rev. B 52 (1995).

${ }^{15}$ C. C. Chen and Y. C. Chou, Phys. Rev. Lett. 54 (1985).

${ }^{16} \mathrm{G}$. Genolet, PhD thesis, EPFL, Lausanne, Switzerland, 2001.

${ }^{17}$ H. Yu, O. Balogun, B. Li, T. W. Murray, and X. Zhang, MEMS, 654 (2005). 